\title{
Improved Genome Sequence and Gene Annotation Resource for the Potato Late Blight Pathogen Phytophthora infestans
}

\author{
Yoonyoung Lee, ${ }^{1}$ Kwang-Soo Cho, ${ }^{2, \dagger}$ Jin-Hee Seo, ${ }^{2}$ Kee Hoon Sohn, ${ }^{1,3, \dagger}$ and \\ Maxim Prokchorchik ${ }^{1, \dagger}$ \\ ${ }^{1}$ Department of Life Sciences, Pohang University of Science and Technology, Pohang 37673, Republic \\ of Korea \\ ${ }^{2}$ Potato Research Team, Highland Agriculture Research Institute, Rural Development Administration, \\ Gangwon 25342, Republic of Korea \\ ${ }^{3}$ School of Interdisciplinary Bioscience and Bioengineering, Pohang University of Science and \\ Technology, Pohang 37673, Republic of Korea
}

\begin{abstract}
Phytophthora infestans is a devastating pathogen causing potato late blight (Solanum tuberosum). Here we report the sequencing, assembly and genome annotation for two Phytophthora infestans isolates sampled in Republic of Korea. Genome sequencing was carried out using long read (Oxford Nanopore) and short read (lllumina Nextseq) sequencing technologies that significantly improved the contiguity and quality of $P$. infestans genome assembly. Our resources would help researchers better understand the molecular mechanisms by which $P$. infestans causes late blight disease in the future.
\end{abstract}

\section{Genome Announcement}

Phytophtora infestans is one of the major threats to the potato and tomato industry all over the world. This oomycete pathogen causes late blight in potato and tomato and it has been a serious concern throughout the history of humankind, with the most damaging outbreak resulting in the Irish potato famine (Bourke et al. 1993; Fry 2016). Due to its scientific and economic importance, $P$. infestans has been extensively studied. Recently, the research focus has been to better understand the coevolution between $P$. infestans and its host plants at the molecular level (Kamoun et al. 2015; Leesutthiphonchai et al. 2018).

Similar to other plant pathogens, $P$. infestans is expected to deliver many effector proteins into the host plant cells and intercellular space. The main function of these effector proteins is to suppress plant immunity responses and promote pathogen proliferation in the host tissue (Hogenhout et al. 2009; Kamoun 2006). Most $P$. infestans effectors carry N-terminal signal peptide sequences for secretion (Haas et al. 2009; Kamoun 2006). In addition, many P. infestans effector

${ }^{\dagger}$ Corresponding authors. M. Prokchorchik; prokchorchik@postech.ac.kr,

K. H. Sohn; khsohn@postech.ac.kr, and K.-S. Cho; kscholove@korea.kr

Current address for Kwang-Soo Cho: Upland Crop Breeding Research Division, Rural Development Administration, Miryang 50424, Republic of Korea

Yoonyoung Lee and Kwang-Soo Cho contributed equally.

*The $e$-Xtra logo stands for "electronic extra" and indicates that a supplementary table is published online.

The author(s) declare no conflict of interest.

Accepted for publication 18 April 2020.

(C) 2020 The American Phytopathological Society

\section{Funding}

This work was carried out with the support of Rural Development Administration, Republic of Korea "Cooperative Research Program for Agriculture Science \& Technology Development (project number PJ01418603)".

\section{Keywords}

genomics, potato late blight, Phytophthora infestans,

Solanum tuberosum 
proteins carry a RXLR or crinkler (CRN, for crinkling and necrosis; LXLFLAK) motif required for translocation into host cell (Zhang et al. 2016). Interestingly, some of these effectors can be recognized by specific intracellular NLR (nucleotide-binding domain leucine-rich repeatcontaining) receptors and activate plant immunity (Birch et al. 2008; Morgan and Kamoun 2007).

In order to gain insights into $P$. infestans epidemiology and its interaction with host plants, several genome studies have been conducted. It was found that the $P$. infestans genome is the largest of all sequenced species of the Phytophthora genus and a significant part of it (approximately $74 \%$ ) consists of repetitive sequence (Haas et al. 2009). Such high repeat content is not only the evidence for genome expansion under a strong evolutionary pressure but also a significant obstacle for reliable genome assembly (Gijzen 2009; Thomma et al. 2016). The current $P$. infestans reference genome has been generated by Sanger-based random shotgun sequencing (Haas et al. 2009). In addition, the short read-based draft genome assemblies of multiple Phytophthora species have been reported (Liu et al. 2016; Longmuir et al. 2017; Reyes-Tena et al. 2019; Studholme et al. 2019; Vetukuri et al. 2018). Recently, several long-read sequencing-aided high contiguity genome assemblies of Phytophthora ramorum (Malar C et al. 2019a and b), P. capsici (Cui et al. 2019), P. cactorum (Yang et al. 2018), and $P$. sojae (Fang et al. 2020) became available. Utilizing the long-read sequencing technologies was shown to dramatically improve the assembly contiguity, especially around the highly repetitive DNA sequences. Therefore, we used the combination of long- and short-read sequencing technologies in order to achieve high contiguity and accuracy of genome assembly for one of the most important plant pathogens, Phytophthora infestans (Kamoun et al. 2015).

In the Republic of Korea, four major clonal lineages, KR_1_A1, KR_2_A2, SIB-1, and US-11, have been identified by genotyping with 12 simple sequence repeat (SSR) markers. KR_1_A1 and KR_2_A2 are Korea-specific genotypes. KR_1_A1, which is predominant in the Korean peninsula, is sensitive to metalaxyl and is the A1 mating type. KR_2_A2 is highly resistant to metalaxyl and is the A2 mating type found in Miryang, where winter cultivation of potato is common (Choi et al. 2020).

Two Phytophthora infestans isolates KR_1_A1 and KR_2_A2 were isolated from diseased potato plants in Daegwallyeong and Miryang cities, respectively, in the Republic of Korea. To prepare mycelia, $P$. infestans isolates were grown on V8 medium ( $17 \mathrm{~g}$ of agar, $50 \mathrm{mg}$ of $\beta$-sistosterol, $900 \mathrm{ml}$ of distilled water, $100 \mathrm{ml}$ of V8 juice, $1.5 \mathrm{~g}$ of calcium carbonate) for 7 days. To increase mycelia production, each isolate was inoculated to $10 \mathrm{ml}$ of liquid pea medium (120 g of frozen peas, 1 liter of distilled water) and was incubated for 14 days in a shaking incubator at $18^{\circ} \mathrm{C}$ and $160 \mathrm{rpm}$. Subsequently, mycelia were collected and were freeze-dried for DNA isolation. To prepare genomic DNA extraction for Oxford Nanopore Technology (ONT) sequencing, GeneAll Exgene Plant SV mini kit (GeneAll) was used for genomic DNA extraction according to manufacturer instructions. Genomic DNA libraries for ONT sequencing were constructed using the ONT 1D ligation sequencing kit, according to the manufacturer instructions. Sequencing was carried out using a single 1D flowcell for each isolate. To prepare genomic DNA extraction for Illumina sequencing, the fungi/yeast genomic DNA isolation kit (Norgen Corp.) was used, according to manufacturer instructions. Then, genomic DNA libraries with an average insert size of $350 \mathrm{bp}$ were constructed according to the standard Illumina TruSeq Nano library protocol.

Phytophthora infestans KR_1_A1 isolate genomic DNA was sequenced using ONT Gridlon and Illumina Nextseq 2000 technologies, providing $63 \times$ long-read and $35 \times$ short-read genome coverage, respectively. Illumina short reads were quality-filtered and adapters were trimmed using fastp v0.19.7 (Chen et al. 2018). Long ONT reads were corrected using Canu v1.8 (Koren et al. 2017) correct command with the following settings, which resulted in $48 \times$ corrected read genome coverage: corOutCoverage $=500$ corMinCoverage $=2$ minReadLength $=2000$ genomeSize $=250 \mathrm{~m}$. The corrected reads were assembled using SMARTdenovo v1.0, with - $\mathrm{k} 17$, -c 1 preferences, to generate the long-read preassembly. The preassembly was iteratively polished using the Illumina short reads. For this, read mapping was performed using bwa-mem v0.7.17 (Li and Durbin 2009) and polishing was carried out using Pilon v1.23 (Walker et al. 2014). Each polishing iteration was followed by the genome completeness assessment, using BUSCO v3 (Simão et al. 2015) with protists set, until the BUSCO score did not improve with the following polishing iteration. The final assembly BUSCO score was $99.1 \%$ with 213 complete BUSCO ortholog groups presented of a total 215 in the Protista set (Table 1).

1026 / Molecular Plant-Microbe Interactions 
Table 1. Genome assembly and gene prediction statistics for Phytopthora infestans isolates

\begin{tabular}{|c|c|c|c|}
\hline Parameters & T30-4 & KR_1_A1 & KR_2_A2 \\
\hline Genome assembly size (bp) & $228,543,505$ & $200,673,608$ & $230,945,478$ \\
\hline Number of contigs & 18,288 & 1,510 & 3,344 \\
\hline Contig N50 (bp) & 44,484 & 291,816 & 154,690 \\
\hline Longest contig (bp) & 582,831 & $1,705,040$ & $1,781,277$ \\
\hline Spanned gaps (bp) & $38,410,029$ & 0 & 1,700 \\
\hline Repeats (\%) & 74 & 72.2 & 71.99 \\
\hline Total genes predicted & 17,797 & 20,172 & 23,771 \\
\hline Number of RXLR effectors ${ }^{a}$ & 306 & 433 & 310 \\
\hline Number of CRN effectors ${ }^{a}$ & 54 & 40 & 50 \\
\hline Completeness (BUSCO score) & $96.3 \%$ & $99.1 \%$ & $98.6 \%$ \\
\hline
\end{tabular}

a RXLR and CRN effectors for all isolates were predicted in this study using EffectR and SignalP5.

KR_2_A2 genome sequencing yielded 49x long-read and 30x short-read genome coverages (Supplementary Table S1). However, after Canu read correction, the long-read $\mathrm{N}_{50}$ length for KR_2_A2 was 5,579 bp, in contrast to 8,226 bp for KR_1_A1. We could not achieve satisfying results with Canu and SMARTdenovo assembly in terms of genome completeness and contiguity. Therefore, we used MaSuRCA v3.3.1 (Zimin et al. 2013), which polishes long reads with Illumina data prior to assembly, to generate the KR_2_A2 genome. The final assembly BUSCO score for KR_2_A2 was 98.6\%, with 212 complete BUSCO ortholog groups presented of a total 215 in the Protista set (Table 1).

We further predicted gene models for $P$. infestans KR_1_A1 and KR_2_A2 isolates using the FunGAP gene annotation pipeline (Min et al. 2017). We utilized the transcriptome data available for $P$. infestans T30-4 (Short Read Archive accession SRS3443412) to aid our gene model prediction, since its read alignment rates were $92.3 \%$ and $92.33 \%$ for KR_1_A1 and KR_2_A2, respectively. We could predict a total of 20,172 genes, for KR_1_A1, and 23,771 genes, for KR_2_A2, which is significantly more than the 17,797 genes predicted in the previous $P$. infestans genome release (Haas et al. 2009). This difference is likely due to the newly discovered sequences that were previously reported as gaps and the use of different gene annotation tools. Next, we set out to identify the RXLR and CRN effector genes in the genomes of Korean $P$. infestans isolates. We first generated the six-frame translation of the T30-4 reference isolate and discarded any open reading frame (ORF) less than $100 \mathrm{bp}$ in length. The resulting ORFs were used as input data for the effectR (Tabima and Grünwald 2019) tool to predict RXLR and CRN effectors. Subsequently, the RXLR and CRN candidate effectors were further analyzed for the presence of eukaryotic signal peptide, using SignalP5 (Almagro Armenteros et al. 2019). We have adjusted the SignalP5 sensitivity threshold to 0.7 as, in the same condition, functionally validated $P$. infestans effectors including PexRD36, PexRD1, Avrblb1, Avr4, and Avr3a were identified (Tabima and Grünwald 2019). As a result, we identified a total of $306 \mathrm{RXLR}$ and $54 \mathrm{CRN}$ effectors in the reference T30-4 genome. These numbers are smaller than what were reported previously (Tabima and Grünwald 2019), likely due to the stringent SignalP5 threshold used in this study. In the same conditions, we identified 433 RXLR and 40 CRN effector genes in KR_1_A1 and 310 RXLR and 50 CRN effector genes in KR_2_A2 genomes. Interestingly, we could identify significantly more effector candidates from KR_1_A1 than KR_2_A2 genome. We believe that the discrepancy was caused by a difference in the contiguity of genome assemblies of the two isolates.

Full genome assembly data and genome annotations were deposited to the National Center for Biotechnology Information database under BioProject accession number PRJNA592863.

\section{Author-Recommended Internet Resource}

GitHub SMARTdenovo v1.0: https://github.com/ruanjue/smartdenovo 


\section{Literature Cited}

Almagro Armenteros, J. J., Tsirigos, K. D., Sønderby, C. K., Petersen, T. N., Winther, O., Brunak, S., von Heijne, G., and Nielsen, H. 2019. SignalP 5.0 improves signal peptide predictions using deep neural networks. Nat. Biotechnol. 37: 420-423.

Birch, P. R., Boevink, P. C., Gilroy, E. M., Hein, I., Pritchard, L., and Whisson, S. C. 2008. Oomycete RXLR effectors: Delivery, functional redundancy and durable disease resistance. Curr. Opin. Plant Biol. 11:373-379.

Bourke, A., Hill, J. R., and Gráda, C. Ó. 1993. The Visitation of God? The Potato and the Great Irish Famine. Lilliput Press, Limited, Dublin, Ireland.

Chen, S., Zhou, Y., Chen, Y., and Gu, J. 2018. fastp: An ultra-fast all-in-one FASTQ preprocessor. Bioinformatics 34:1884-1890.

Choi, J.-G., Hong, S.-Y., Kessel, G.J.T., Cooke, D.E.L., Vossen, J.H., Cho, J.-H., Im, J.-S., Park, Y.-E., and Cho, K.-S. 2020. Genotypic and phenotypic characterization of Phytophthora infestans in South Korea during 2009-2016 reveals clonal reproduction and absence of EU_13_A2 genotype. Plant Pathol. 69: 932-943.

Cui, C., Herlihy, J. H., Bombarely, A., McDowell, J. M., and Haak, D. C. 2019. Draft assembly of Phytophthora capsici from long-read sequencing uncovers complexity. Mol. Plant-Microbe Interact 32:1559-1563.

Fang, Y., Coelho, M. A., Shu, H., Schotanus, K., Thimmappa, B. C., Yadav, V., Chen, H., Malc, E. P., Wang, J., Mieczkowski, P. A., Kronmiller, B., Tyler, B. M., Sanyal, K., Dong, S., Nowrousian, M., and Heitman, J. 2020. Long transposon-rich centromeres in an oomycete reveal divergence of centromere features in Stramenopila-Alveolata-Rhizaria lineages. PLoS Genet. 16:e1008646.

Fry, W. E. 2016. Phytophthora infestans: New tools (and old ones) lead to new understanding and precision management. Annu. Rev. Phytopathol. 54:529-547.

Gijzen, M. 2009. Runaway repeats force expansion of the Phytophthora infestans genome. Genome Biol. 10:241.

Haas, B. J., Kamoun, S., Zody, M. C., Jiang, R. H. Y., Handsaker, R. E., Cano, L. M., Grabherr, M., Kodira, C. D., Raffaele, S., Torto-Alalibo, T., Bozkurt, T. O., AhFong, A. M. V., Alvarado, L., Anderson, V. L., Armstrong, M. R., Avrova, A., Baxter, L., Beynon, J., Boevink, P. C., Bollmann, S. R., Bos, J. I. B., Bulone, V., Cai, G., Cakir, C., Carrington, J. C., Chawner, M., Conti, L., Costanzo, S., Ewan, R., Fahlgren, N., Fischbach, M. A., Fugelstad, J., Gilroy, E. M., Gnerre, S., Green, P. J., Grenville-Briggs, L. J., Griffith, J., Grünwald, N. J., Horn, K., Horner, N. R., Hu, C.-H., Huitema, E., Jeong, D.-H., Jones, A. M. E., Jones, J. D. G., Jones, R. W., Karlsson, E. K., Kunjeti, S. G., Lamour, K., Liu, Z., Ma, L., Maclean, D., Chibucos, M. C., McDonald, H., McWalters, J., Meijer, H. J. G., Morgan, W., Morris, P. F., Munro, C. A., O'Neill, K., Ospina-Giraldo, M., Pinzón, A., Pritchard, L., Ramsahoye, B., Ren, Q., Restrepo, S., Roy, S., Sadanandom, A., Savidor, A., Schornack, S., Schwartz, D. C., Schumann, U. D., Schwessinger, B., Seyer, L., Sharpe, T., Silvar, C., Song, J., Studholme, D. J., Sykes, S., Thines, M., van de Vondervoort, P. J. I., Phuntumart, V., Wawra, S., Weide, R., Win, J., Young, C., Zhou, S., Fry, W., Meyers, B. C., van West, P., Ristaino, J., Govers, F., Birch, P. R. J., Whisson, S. C., Judelson, H. S., and Nusbaum, C. 2009. Genome sequence and analysis of the Irish potato famine pathogen Phytophthora infestans. Nature 461:393-398.

Hogenhout, S. A., Van der Hoorn, R. A., Terauchi, R., and Kamoun, S. 2009. Emerging concepts in effector biology of plant-associated organisms. Mol. PlantMicrobe Interact 22:115-122.

Kamoun, S. 2006. A catalogue of the effector secretome of plant pathogenic oomycetes. Annu. Rev. Phytopathol. 44:41-60.

Kamoun, S., Furzer, O., Jones, J. D., Judelson, H. S., Ali, G. S., Dalio, R. J., Roy, S. G., Schena, L., Zambounis, A., Panabières, F., Cahill, D., Ruocco, M., Figueiredo, A., Chen, X. R., Hulvey, J., Stam, R., Lamour, K., Gijzen, M., Tyler, B. M., Grünwald, N. J., Mukhtar, M. S., Tomé, D. F., Tör, M., Van Den Ackerveken, G., McDowell, J., Daayf, F., Fry, W. E., Lindqvist-Kreuze, H., Meijer, H. J., Petre, B., Ristaino, J., Yoshida, K., Birch, P. R., and Govers, F. 2015. The Top 10 oomycete pathogens in molecular plant pathology. Mol. Plant Pathol. 16: 413-434.
Koren, S., Walenz, B. P., Berlin, K., Miller, J. R., Bergman, N. H., and Phillippy, A. M. 2017. Canu: Scalable and accurate long-read assembly via adaptive $k$-mer weighting and repeat separation. Genome Res. 27:722-736.

Leesutthiphonchai, W., Vu, A. L., Ah-Fong, A. M. V., and Judelson, H. S. 2018. How does Phytophthora infestans evade control efforts? Modern insight into the late blight disease. Phytopathology 108:916-924.

Li, H., and Durbin, R. 2009. Fast and accurate short read alignment with BurrowsWheeler transform. Bioinformatics 25:1754-1760.

Liu, H., Ma, X., Yu, H., Fang, D., Li, Y., Wang, X., Wang, W., Dong, Y., and Xiao, B. 2016. Genomes and virulence difference between two physiological races of Phytophthora nicotianae. Gigascience 5:3.

Longmuir, A. L., Beech, P. L., and Richardson, M. F. 2017. Draft genomes of two Australian strains of the plant pathogen, Phytophthora cinnamomi. F1000 Res. 6 : 1972.

Malar C, M., Yuzon, J. D., Das, S., Das, A., Panda, A., Ghosh, S., Tyler, B. M., Kasuga, T., and Tripathy, S. 2019b. Haplotype-phased genome assembly of virulent Phytophthora ramorum isolate ND886 facilitated by long-read sequencing reveals effector polymorphisms and copy number variation. Mol. PlantMicrobe Interact 32:1047-1060

Malar C, M., Yuzon, J. D., Panda, A., Kasuga, T., and Tripathy, S. 2019a. Updated assembly of Phytophthora ramorum pr102 isolate incorporating long reads from PacBio sequencing. Mol. Plant-Microbe Interact 32:1472-1474.

Min, B., Grigoriev, I. V., and Choi, I. G. 2017. FunGAP: Fungal genome annotation pipeline using evidence-based gene model evaluation. Bioinformatics 33 : 2936-2937.

Morgan, W., and Kamoun, S. 2007. RXLR effectors of plant pathogenic oomycetes. Curr. Opin. Microbiol. 10:332-338.

Reyes-Tena, A., Huguet-Tapia, J. C., Lamour, K. H., Goss, E. M., RodríguezAlvarado, G., Vázquez-Marrufo, G., Santillán-Mendoza, R., and FernándezPavía, S. P. 2019. Genome sequence data of six isolates of Phytophthora capsici from Mexico. Mol. Plant-Microbe Interact 32:1267-1269.

Simão, F. A., Waterhouse, R. M., Ioannidis, P., Kriventseva, E. V., and Zdobnov, E. M. 2015. BUSCO: Assessing genome assembly and annotation completeness with single-copy orthologs. Bioinformatics 31:3210-3212.

Studholme, D. J., Panda, P., Sanfuentes Von Stowasser, E., González, M., Hill, R., Sambles, C., Grant, M., Williams, N. M., and McDougal, R. L. 2019. Genome sequencing of oomycete isolates from Chile supports the New Zealand origin of Phytophthora kernoviae and makes available the first Nothophytophthora sp. genome. Mol. Plant Pathol. 20:423-431.

Tabima, J. F., and Grünwald, N. J. 2019. effectR: An expandable R package to predict candidate RxLR and CRN Effectors in oomycetes using motif searches. Mol. Plant-Microbe Interact 32:1067-1076.

Thomma, B. P. H. J., Seidl, M. F., Shi-Kunne, X., Cook, D. E., Bolton, M. D., van Kan, J. A. L., and Faino, L. 2016. Mind the gap; seven reasons to close fragmented genome assemblies. Fungal Genet. Biol. 90:24-30.

Vetukuri, R. R., Kushwaha, S. K., Sen, D., Whisson, S. C., Lamour, K. H., and Grenville-Briggs, L. J. 2018. Genome sequence resource for the oomycete Taro pathogen Phytophthora colocasiae. Mol. Plant-Microbe Interact 31:903-905.

Walker, B. J., Abeel, T., Shea, T., Priest, M., Abouelliel, A., Sakthikumar, S., Cuomo, C. A., Zeng, Q., Wortman, J., Young, S. K., and Earl, A. M. 2014. Pilon: An integrated tool for comprehensive microbial variant detection and genome assembly improvement. PLoS One 9:e112963.

Yang, M., Duan, S., Mei, X., Huang, H., Chen, W., Liu, Y., Guo, C., Yang, T., Wei, W. Liu, X., He, X., Dong, Y., and Zhu, S. 2018. The Phytophthora cactorum genome provides insights into the adaptation to host defense compounds and fungicides. Sci. Rep. 8:6534.

Zhang, D., Burroughs, A. M., Vidal, N. D., Iyer, L. M., and Aravind, L. 2016. Transposons to toxins: The provenance, architecture and diversification of a widespread class of eukaryotic effectors. Nucleic Acids Res. 44:3513-3533.

Zimin, A. V., Marçais, G., Puiu, D., Roberts, M., Salzberg, S. L., and Yorke, J. A. 2013. The MaSuRCA genome assembler. Bioinformatics 29:2669-2677. 